\section{La cuestión del cuerpo en el campo de la comunicación. Pensando aportes y desafíos a partir del caso del Área Cuerpo y Comunicación de la Universidad de Buenos Aires}

The question of the body in the field of communication. Thinking contributions and challenges from the case of the Body and Communication Area, University of Buenos Aires

https://doi.org/10.18566/comunica.n44.a06

Recibido: 15 de febrero de 2021

Aceptado: 25 de marzo de 2021

\section{Resumen}

En los últimos años, el interés por la cuestión del cuerpo se ha visto reforzado en el campo de la comunicación. La masificación de los feminismos y las transformaciones estético-sensibles de la última década han profundizado desplazamientos epistemológicos tendientes a conceder mayor relevancia a la problemática de la corporalidad y la comunicación entre cuerpos. La valorización del cruce "cuerpo y comunicación" se expresa en la apertura de espacios institucionales, en la elección de objetos de estudio en tesis de grado y posgrado, y en desarrollos curriculares.

No obstante, el registro y tematización de los avances al respecto resulta aún incipiente. El objetivo del presente texto es analizar los aportes del cruce "cuerpo y comunicación" al campo de la comunicación, enfocándose en el abordaje de las actividades y temáticas desarrolladas en un espacio institucional específico: el "Área Cuerpo y Comunicación. Problemáticas comunicación

número 44

Enero - junio

2021 | pp. 93-110

\section{Dra. Mariela Singer}

Doctora en Ciencias Sociales y licenciada en Ciencias

de la Comunicación por la Universidad de Buenos Aires (UBA). Docente de grado y de posgrado en la Facultad de Arquitectura, Diseño y Urbanismo y en la Facultad de Ciencias Sociales de la misma institución. Directora del Grupo de Investigación en Comunicación "Cuerpo, Comunicación, Estética y Política" (GIC 2020-21) y coordinadora del "Área Cuerpo y Comunicación. Problemáticas estéticopolíticas en la producción de subjetividad"; además, profesora adjunta integrante del Circuito de Formación Feminista de la Facultad de Ciencias Sociales (CIRFFEM), jefa de trabajos prácticos de la materia Cuerpo, Imagen y Sentido y profesora adjunta a cargo del seminario curricular de grado Cuerpo, Comunicación y Política en la Producción de Subjetividad (UBA). https: / / orcid.org/00000002-0859-817X marielasing@hotmail.com 
estético-políticas en la producción de subjetividad", de la Universidad de Buenos Aires. Con ese objetivo, el escrito comienza exponiendo desplazamientos y luchas feministas de las últimas décadas que han redundado en la valorización de la corporalidad. En segundo orden, da cuenta de la creación y el avance del área de investigación referida y de los seminarios de grado vinculados a ella. En tercer término, sintetiza las líneas de trabajo desplegadas en el área, para dar cuenta así de aportes del cruce “cuerpo y comunicación” al campo de la comunicación.

Finalmente, reflexiona sobre el crecimiento de la temática del cuerpo y los desplazamientos de los últimos años, para concluir que entre los principales desafios de este campo está generar aperturas curriculares que permitan integrar los aportes referidos. El trabajo se enmarca en una metodología cualitativa de enfoque autoetnográfico y se basa en la recuperación de técnicas de "participación observante", registros personales y análisis de documentos institucionales.

\section{Abstract}

In recent years, interest in the question of the body has been reinforced in the field of communication. The massification of feminisms and the aesthetic-sensitive transformations of the last decade have deepened epistemological displacements that tend to give greater relevance to the problem of corporality and communication between bodies. The valorization of the intersection "body and communication" is expressed in the opening of institutional spaces, in the choice of objects of study in undergraduate and graduate thesis, and in curricular developments.

However, the recording and thematization of progress in this regard is still incipient. The objective of this text is to analyze the contributions of the "body and communication" intersection to the field of communication, focusing on the approach to activities and themes developed in a specific institutional space: the "Research Area Body and Communication. Aestheticpolitical problems in the production of subjectivity", University of Buenos Aires, Argentina. Following this objective, the writing begins by reviewing feminist displacements and struggles of the last decades that have resulted in the valorization of corporality. Secondly, it outlines the creation and progress of the referred research area and the degree seminars linked to it. Third, it synthesizes the lines of work deployed in the area, thus outlining the contributions of the "body and communication" intersection to the field of communication.

Finally, it reflects on the growth of the subject of the body and displacements in recent years, concluding that among the main challenges in the field is that of generating curricular openings that allow integrating the referred

\section{Palabras clave}

Cuerpo, comunicación, feminismos, aportes, campo.

\section{Key words}

Body, communication, feminisms, contributions, field. 
contributions. The work is framed in a qualitative methodology with an autoethnographic approach and is based on the recovery of techniques of "observant participation", personal records and analysis of institutional documents.

\section{Introducción}

En los últimos años, el interés por la cuestión del cuerpo se ha visto reforzado en el campo de la comunicación. La masificación de los feminismos y las transformaciones en las subjetividades y sensibilidades han redundado en desplazamientos en el terreno académico, en el cual, frente a tradiciones dualistas, racionalistas y androcéntricas, tiende a concederse mayor relevancia a la cuestión de la corporalidad (Gago, 2020; Bardet, 2018, p. 24). En el campo de la comunicación, específicamente, el interés por el cuerpo supone también un desplazamiento hacia la preocupación por pensar aquella sin reducirla a fenómenos mediáticos y la apertura de una zona problemática vinculada al abordaje de los encuentros entre cuerpos, ya sea en experiencias artísticas, del activismo u otros ámbitos.

Las redes de afecto entre cuerpos en instancias asamblearias o manifestaciones políticas, o incluso en complementariedad con el activismo "tecnopolítico" (Fuentes, 2015, 2020), la vivencia intensa compartida entre dos cuerpos en una danza, las relaciones sexo-afectivas y los lazos intersubjetivos cotidianos, entre muchos otros, son fenómenos que interpelan a quienes forman parte del campo con la sospecha de que en la perspectiva comunicacional hay una potencia singular para abordarlos y de que la pregunta por la "comunicación" producida entre los cuerpos puede aportar desarrollos específicos.

En síntesis, en los últimos años se evidencia el convencimiento, cada vez más afianzado, de que es necesario desarmar la asimilación frecuente entre "comunicación" y "fenómenos mediáticos" (Rizo García, 2018, p. 3), y de que la comunicación entre cuerpos, así como la pregunta por las modalidades de encuentro involucradas, resultan ineludibles para pensar la producción de subjetividad. Esta preocupación creciente ha tendido a hacer que se conciba en los últimos años el cruce "cuerpo y comunicación" como términos indisociables que, en conjunto, delimitan una zona problemática específica no cubierta en otros campos.

Así, la valorización de la problemática "cuerpo y comunicación” resulta cada vez más expresa y se manifiesta en la apertura de espacios institucionales, 
en la elección de objetos de estudio de tesis de grado y posgrado, y en desarrollos curriculares. No obstante, el registro y tematización de los avances al respecto resulta aún incipiente (Rizo García, 2018, p. 9; Cáneva, 2020, pp. 13-14).

El objetivo del presente texto es analizar los aportes del cruce "cuerpo y comunicación" al campo de la comunicación desarrollados en un espacio institucional específico de reconocimiento en el campo: el "Área Cuerpo y Comunicación. Problemáticas estético-políticas en la producción de subjetividad" de la Universidad de Buenos Aires. Para cumplir ese objetivo, el escrito comienza exponiendo desplazamientos de las últimas décadas y luchas feministas de los últimos años que han redundado en la valorización de la corporalidad. En segundo orden, da cuenta de la creación y el avance del área de investigación referida y de los seminarios de grado vinculados a ella. Tercero, sintetiza las líneas de trabajo desplegadas en el área y en los seminarios vinculados a su marco, dando cuenta así de aportes del cruce "cuerpo y comunicación" al campo de la comunicación. Finalmente, reflexiona sobre el crecimiento de la temática del cuerpo en el campo, sobre los desplazamientos hacia nuevas preocupaciones en los últimos años y sobre los principales desafíos para posibilitar la incorporación sistematizada de esos aportes y preocupaciones.

El trabajo se enmarca en un enfoque metodológico autoetnográfico, consistente en una estrategia cualitativa que valoriza la propia experiencia y puesta en juego del cuerpo del investigador en el fenómeno estudiado (Scribano y De Sena, 2009, p. 3). En este marco, como fuentes de relevamiento particulares para el presente texto se incluyeron técnicas de "participación observante" (Aschieri y Puglisi, 2010, p. 128), registros personales, indagación bibliográfica y análisis de documentos institucionales. Se contó con el acceso consentido de las autoras y los autores de los trabajos monográficos entregados en los seminarios vinculados al área, así como de los integrantes y las integrantes del área para el registro de las actividades desarrolladas en este espacio institucional.

\section{Desplazamientos hacia la valorización de lo sensible y de los cuerpos en las últimas décadas}

En las últimas décadas, especialmente a partir de la segunda mitad del siglo XX, y sobre todo de la década del 60, han operado desplazamientos tendientes a valorizar lo sensible y la corporalidad frente a tradiciones racionalistas. A la vez, estos desplazamientos se han agudizado en los últimos años con la masificación de los feminismos. 
Los años 60 y 70 configuran un período de radicalización política y estética que condensa diversidad de experiencias y procesos: las demandas de derechos civiles y el activismo negro; el antibelicismo; el movimiento hippie; los movimientos estudiantiles; la experimentación con la percepción; el rock e roll; los feminismos y las proclamas por la liberación del cuerpo; la exploración de relaciones sexo-afectivas y lazos comunitarios, entre varios otros. En este marco se afianzan formas de pensar la política cuestionadoras de verdades universales y de "grandes relatos" que asumen como objeto de crítica cuestiones micropolíticas, incluyendo la problematización de las jerarquías en el hogar, la academia y el partido. Asimismo, es un período de valorización de la experimentación, de la sensibilidad y la afectividad frente a lógicas tradicionalmente racionalistas, y de ponderación de saberes manuales y experienciales frente a saberes teóricos o expertos.

A partir de los años 60, y pronunciándose en la década de los 70, se produce un impresionante renacer de los movimientos feministas, con especial fuerza en Estados Unidos, que pone el acento en la sexualidad, la familia, el trabajo, los derechos reproductivos y los cuerpos. El lema "Lo personal es político" surgido en esos años (en lo que se conoce como "la segunda ola" del feminismo anglosajón, luego de una "primera ola" caracterizada por las demandas sufragistas) resume el espíritu de buena parte de las proclamas, que incluyen la problematización de las relaciones informales en ámbitos cotidianos y de las desigualdades puestas en acto en la sexualidad, los vínculos familiares y los afectos. En este segundo momento, las demandas no apuntan primordialmente a la consecución de políticas formales (en las que se había centrado la "primera ola"), sino que problematizan con la misma fuerza las prácticas micropolíticas.

Por otro lado, en ese contexto generalizado de interés por la experimentación, la corporalidad y la sensibilidad de los años 60 y 70, los feminismos ejercen un rol significativo en la valorización del cuerpo promoviendo el pensamiento de este como territorio político.

Como plantea la reconocida pensadora feminista Silvia Federici (2015), las activistas y teóricas feministas han visto históricamente en el concepto de "cuerpo" una clave para comprender las raíces del dominio masculino y de la construcción de la identidad social feminizada. Buena parte del discurso feminista sobre el cuerpo se orienta a subvertir la identificación de las mujeres con una versión racionalista y degradada del cuerpo, solidaria a la jerarquización de las facultades humanas: "La degradación de la realidad corporal ha sido históricamente instrumental a la consolidación del poder patriarcal y a la explotación masculina del trabajo femenino" (2015, p. 25). Así, subraya la autora: 
La enorme cantidad de estudios feministas que se han producido desde principios de los años setenta acerca del control ejercido sobre la función reproductiva de las mujeres, los efectos de las violaciones y el maltrato y la imposición de la belleza como una condición de aceptación social constituyen una enorme contribución al discurso sobre el cuerpo en nuestros tiempos (...). Partiendo de un análisis de la "política del cuerpo", las feministas no sólo han revolucionado el discurso filosófico y político contemporáneo, sino que también han comenzado a revalorizar el cuerpo (2015, p. 25).

La también teórica feminista Rosi Braidotti (2004) subraya la valorización del cuerpo producida en los feminismos: "En el marco conceptual feminista el sitio primario de localización es el cuerpo. El sujeto no es una entidad abstracta, sino material incardinada o corporizada" (2004, p. 16). A la vez, remarca que esto no implica que el cuerpo deba pensarse como una esencia o naturaleza, al contrario: "El cuerpo no es una cosa natural, por el contrario, es una entidad socializada, codificada culturalmente" (2004, p. 16). En el mismo sentido, Federici destaca que la valorización del cuerpo en las perspectivas feministas que retoma no se produce bajo la forma de concebirlo como una entidad biológicamente dada, y advierte contra identificar el "retorno al cuerpo" (2015, p. 27) con la recuperación de una naturaleza o esfera privada, deshistorizada y despolitizada. La autora rescata, en cambio, "el saber feminista que se niega a identificar el cuerpo con la esfera de lo privado y, en esa línea, habla de una "política del cuerpo'” (2015, p. 28).

A fines de los años 80, y sobre todo a partir de la década de los 90, los desarrollos de la teoría queery su ingreso en el ámbito académico conforman otro hito de los cambios en el pensamiento feminista y su valorización del cuerpo. Las elaboraciones de pensadoras como Judith Butler (1999) y Donna Haraway (1995), entre otras, profundizan problematizaciones sobre la cuestión del cuerpo cuestionando la discontinuidad implicada entre naturaleza y cultura en concepciones de "género" que, diferenciándolo del sexo, reducían este último a una entidad biológica. Asimismo, las perspectivas queer acentuaban el cuerpo en su materialidad (sin reducir esta a un componente biológico), destacando que "los cuerpos importan" y preguntándose (según la preocupación cada vez mayor por pensar las problemáticas feministas en un cruce interseccional con otras relaciones de explotación) "qué cuerpos importan" (y cuáles no son concebidos como vidas vivibles, dignas o legibles) (Butler, 2002).

Los desplazamientos de las últimas décadas hacia la valorización del cuerpo y los cuerpos han encontrado un nuevo hito en los últimos años a partir de la expansión de los feminismos en el ámbito global. En la Argentina, particularmente, la masificación de los feminismos encuentra como punto de inflexión el año 2015, cuando se llama el 3 de junio de ese año a la primera 
movilización de \#Niunamenos, que logra una convocatoria multitudinaria en las calles. A partir de entonces, otros hitos fueron dando cuenta de la extensión del movimiento, como el primer paro nacional de mujeres el 19 de octubre de 2016; el primer paro internacional de mujeres el 8 de marzo de 2017 (que se replica en un gran número de ciudades y países, y que desde entonces se ha llevado a cabo consecutivamente cada 8 de marzo); y las manifestaciones masivas por la legalización del aborto a partir de 2018 que colman las calles a lo largo de todo el país.

Con su masificación, los feminismos han permeado muy diversos ámbitos y trastocado regímenes sensibles y vínculos sociales. El ámbito académico no es ajeno a estas transformaciones y ha atestiguado desde entonces cambios importantes. La valorización de los cuerpos como territorios políticos de inscripción y transformación del orden social, patente en las luchas feministas de los últimos años, vuelve, por ejemplo, más legibles desarrollos teóricos y conceptuales que desde algunos espacios académicos venían acentuando la importancia política de fenómenos vinculados a la sensibilidad y la corporalidad. Asimismo, las transformaciones referidas han agudizado también el interés por el acercamiento a estos fenómenos de manera notoria en elecciones temáticas de objetos de estudio de trabajos de grado y de posgrado, así como en la apertura de espacios académicos abocados a estas temáticas.

\section{Cuerpo y comunicación. La creación de un área de investigación y formación}

En el terreno de las ciencias sociales y humanidades, la preocupación por el cuerpo es relativamente reciente. En Latinoamérica comenzó a gozar de legitimidad hacia fines de los años 80 y cobró mayor impulso a comienzos del siglo XXI (Rizo García, 2018, p. 1). En el caso de la Argentina, recién a mediados de los 90 se inician estudios sobre corporalidad (Citro, 2010, p. 9). Mayormente, los estudios pioneros son socioantropológicos, y dentro de este marco, desde los años 90, se desarrollan en la línea de la antropología del cuerpo trabajos y espacios de investigación (Citro, 2010, p. 9). A fines de esa década comienzan a desplegarse también desde la sociología espacios y trabajos en la línea de la sociología del cuerpo y las emociones (Scribano, 2016 , p. 1). Por su parte, en la filosofía se ha pronunciado también en los últimos años el interés por la corporalidad y por resquebrajar tradiciones racionalistas, y hace aproximadamente una década confluyó en eventos como el I Congreso Internacional de Filosofías del Cuerpo en la Argentina, realizado en 2013 en Buenos Aires, entre otros. 
En el caso de la comunicación, si bien el interés por la corporalidad se ha agudizado notoriamente en los últimos años, la literatura sobre el avance de la temática en el campo es sumamente escasa (Rizo García, 2018, p. 1) y se vuelve más difícil de delimitar. No obstante, a partir de algunos registros y sistematizaciones de producciones efectuadas en trabajos recientes (por ejemplo, en Scarnatto y De Marziani, 2020), es posible reconocer un despliegue concreto de la problemática del cuerpo en la última década (con antecedentes más dispersos en décadas anteriores), expreso en la apertura de áreas de investigación específicas, seminarios y producción teórica.

En esa dirección, el abordaje del Área Cuerpo y Comunicación en que se concentra este escrito se orienta a contribuir a la circulación y conocimiento del trabajo que se viene realizando en el campo de la comunicación en relación con la cuestión del cuerpo, aportando bibliografía específica sobre un espacio de formación e investigación en "cuerpo y comunicación" de una universidad argentina sumamente reconocida y con gran incidencia en el campo local. Tanto las actividades de intercambio como los seminarios curriculares de grado, además de los grupos de investigación implementados en vinculación con el área $y$, en general, el visible crecimiento de las temáticas de trabajo impulsadas y de las demandas y producciones de estudiantes, investigadoras e investigadores, dan cuenta de un desarrollo significativo que vale la pena registrar y socializar para aportar al avance de la problemática en el campo.

La inauguración del Área Cuerpo y Comunicación se produce en 2013, a cargo de un equipo de docentes y estudiantes que venían trabajando la cuestión del cuerpo en cátedras y ámbitos informales, y deseaban desarrollar un espacio sistematizado de trabajo. Ya desde su inauguración, el área da cuenta del interés por enlazar mundos de lectura y de prácticas corporales (como, por ejemplo, disciplinas de movimiento) en un ámbito académico tradicionalmente excluyente de ese tipo de experiencias; así subraya la relevancia de estudiar no solo los cuerpos, sino "desde" los cuerpos. Se inauguró con una charla informativa y colectiva abierta a la comunidad académica y a otras comunidades del activismo y el arte, y con una actividad conjunta de danza en la que se mezclaron, danzando, docentes, estudiantes, investigadoras, investigadores y participantes de ámbitos ajenos a la universidad.

Desde el inicio, el área se propone como un espacio de investigación conceptual, discusión política y exploración corporal que indaga sobre las modalidades de encuentro entre cuerpos como productoras de subjetividad y afectividad. Plantea la importancia de desbaratar la asimilación frecuente entre "comunicación" y "fenómenos mediáticos", y la necesidad de saldar el vacío en el campo relativo a pensar la comunicación entre cuerpos, que 
constituía a la vez una demanda expresa en la elección temática de las tesinas finales de grado de la carrera de Ciencias de la Comunicación de la institución.

Por otro lado, se propone conformar un espacio de intercambio, formación e investigación en relación con varios ejes: el estudio y sistematización de marcos conceptuales propicios para trabajar el cruce cuerpo y comunicación; la reflexión sobre modalidades de encuentro entre cuerpos en experiencias del arte y el activismo; el estudio de elaboraciones feministas productivas para el abordaje de la cuestión del cuerpo y de la comunicación en vínculos intersubjetivos; y el análisis de políticas de los cuerpos...

Desde su apertura, se promueven actividades regulares de formación e intercambio para trabajar esos ejes, como talleres de lectura quincenales, con la asistencia de estudiantes, docentes, investigadores, investigadoras y artistas de otras casas de estudio vinculadas a prácticas corporales (como la Universidad Nacional de las Artes y la Compañía Nacional de Danza Contemporánea, cuyas y cuyos integrantes participaron también en la organización de actividades del área); exposición de material audiovisual y films sobre las relaciones entre cuerpo, espacio, arquitectura, disciplinas corporales, etc., con charlas posteriores; encuentros de intercambio entre tesistas con problemáticas vinculadas a corporalidad; publicación de materiales en cuadernillos auspiciados por la carrera de Ciencias de la Comunicación (Área Cuerpo y Comunicación, 2015); además de otras actividades, como los encuentros de danza organizados en la facultad, que convocan a intercambiar desde el cuerpo a estudiantes, docentes, investigadores e investigadoras de la institución y a participantes de ámbitos externos.

Ya desde los primeros años, las actividades del área logran una gran convocatoria, y en 2015, la circulación y difusión del espacio aumenta, especialmente gracias a unas notas periodísticas publicadas en diarios masivos nacionales, como Página/12, que rescatan la propuesta y valorizan el intento de "problematizar los modos de implicación entre cuerpo y subjetividad en la universidad" (Torres Cabreros, 2015). Estos medios también publicaron crónicas sobre los encuentros de danza y relataron cómo "los estudiantes se acodan en la baranda que da al patio seco de la Facultad de Ciencias Sociales de la UBA y observan intrigados la actividad", y que "los pies desnudos a veces sobre el piso, a veces sobre el compañero o compañera, a veces cortando el aire de la noche" de quienes bailan y "cómo los cuerpos se entregan al fluir del movimiento conjunto", cuya comunión "desafía con su potencia la lógica hegemónica de la universidad: las relaciones de dominación, el individualismo, la primacía de la razón" (Torres Cabreros, 2015). 

las actividades de encuentro corporal que realiza el área, orientadas estas a enlazar diferentes trayectorias artísticas y académicas, y a mezclar los cuerpos de claustros en una instancia física:

Una masa indefinida de cuerpos brilla a la luz de la luna en el patio de [la Facultad de Cs.] Sociales. A punto de cumplir su segundo año de vida, el Área de Cuerpo y Comunicación de la facultad logró instalar una práctica hasta entonces ajena al ámbito académico (Rubin, 2016).

En ese mismo año (2015), a la vez, el 3 de junio se convoca al primer \#Niunamenos, con una respuesta masiva en las calles que conforma el primer hito de un movimiento que progresivamente se multiplicará e incrementará el interés por temáticas de cuerpos y feminismos, lo que se expresará también en el campo de la comunicación y, particularmente, en el área referida.

En ese año se realiza el Congreso Latinoamericano de Comunicación, llevado a cabo por la carrera de Ciencias de la Comunicación de la UBA a raíz del trigésimo aniversario de su creación. El área participa con actividades que obtienen enorme convocatoria (de hecho, están entre las de mayor concurrencia): con aulas llenas, pedidos de cambios a espacios más grandes para abarcar la cantidad de gente interesada en cuerpo y comunicación, el área logra enorme difusión y continúa aumentando su convocatoria.

Las actividades del congreso incluyen paneles con académicos, académicas, activistas y artistas de gran trayectoria, además de mesas de trabajo con ponencias y un evento final de danza entre las asistentes y los asistentes, estudiantes, docentes, investigadoras e investigadores, que colma el hall central del edificio de la facultad (Imagen 2). Los paneles principales se organizan alreded or de cuatro ejes: "Cuerpo y género", "Cuerpo y experiencias de emancipación”, “Cuerpo y filosofía” y "Cuerpo y danza” (Imagen 1). 
Imagen 1. Flyer de convocatoria a los paneles del Área Cuerpo y Comunicación.

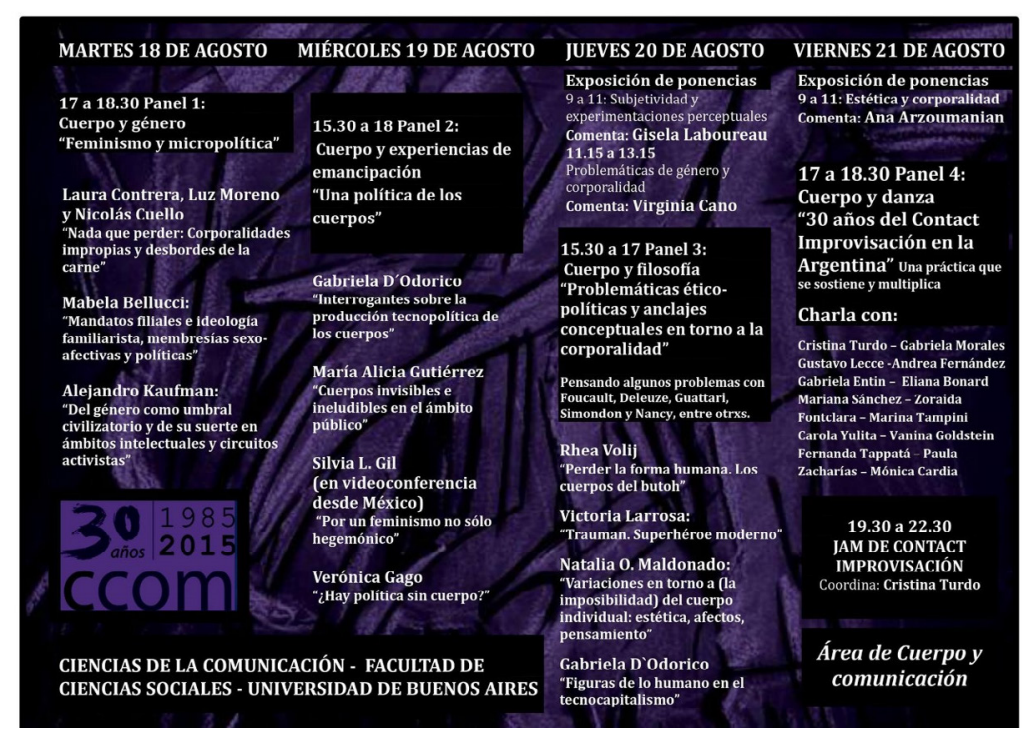

Congreso Latinoamericano de Comunicación, Ciencias de la Comunicación, Facultad de Ciencias Sociales, Universidad de Buenos Aires.

Agosto de 2015.

Imagen 2. Encuentro de danza en la facultad convocado por el Área Cuerpo y Comunicación.

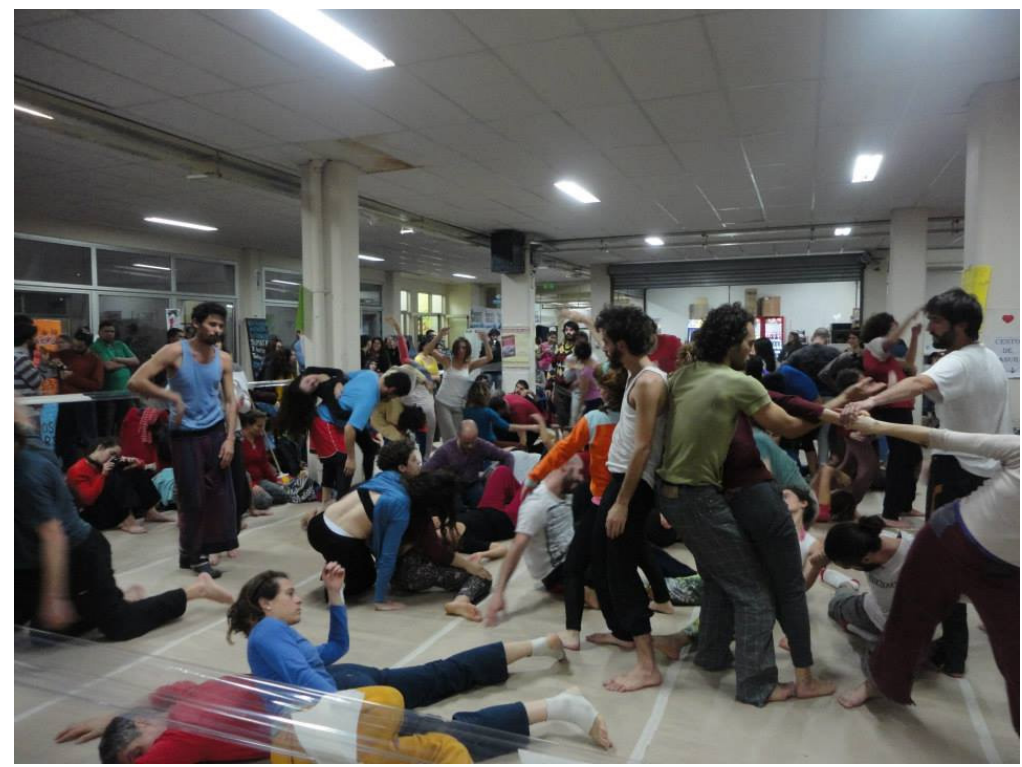

Hall planta baja de la Facultad de Ciencias Sociales, Universidad de Buenos Aires. Agosto de 2015. 
En 2015 se crea también el primer seminario curricular de grado vinculado al área: Cuerpo, Comunicación y Política en la Producción de Subjetividad. Desde su nacimiento, este seminario tiene una gran cantidad de inscritos y en los años siguientes completará y superará ampliamente el cupo (triplicará o cuadriplicará el tope permitido para los seminarios curriculares). En 2019 es integrado al Circuito de Formación Feminista de la Facultad de Ciencias Sociales de la UBA, creado ese mismo año. Desde entonces, el seminario es dictado para todas las carreras de la facultad y logra una demanda mayor cada año (con más de un centenar de personas en 2020).

En 2020 se crea, asimismo y con el área, el Grupo de Investigación en Comunicación "Cuerpo, Comunicación, Estética y Política", dedicado a contener la enorme y creciente demanda de tesistas de Ciencias de la comunicación interesados e interesadas en la cuestión del cuerpo.

\section{Líneas de trabajo en cuerpo y comunicación en el marco del área y los seminarios}

Este apartado sistematiza las principales líneas de trabajo desarrolladas en las actividades y espacios vinculados al Área Cuerpo y Comunicación con base en el análisis documental de las actas del Congreso Latinoamericano de Comunicación (Buenos Aires, 2015), particularmente en los registros del grupo de trabajo 12, que dan cuenta de las ponencias correspondientes al área; en la sistematización de las problemáticas de discusión explícitas en las convocatorias de los talleres de lectura y discusión del área; en el relevamiento y sistematización de las temáticas trabajadas en las monografías finales de los seminarios implementados en el marco del área en los dos últimos años (2019 y 2020); y en el relevamiento de las elecciones de tema de tesinas del Grupo de Investigación en Comunicación.

Como se comentara en la introducción, entre las fuentes de relevamiento están las técnicas de "participación observante" (Aschieri y Puglisi, 2010, p. 128), así como los registros personales y el análisis de los documentos institucionales referidos. Cabe especificar, asimismo, que se contó con el acceso consentido de las autoras y los autores de los trabajos monográficos entregados en los seminarios y para el registro de las actividades desarrolladas en dicho espacio.

En lo que hace a los trabajos del congreso, fueron aceptadas ocho ponencias para integrar las mesas, entre las cuales tres tienen que ver con temáticas de cuerpo y arte (específicamente de danza), y dos más con cuestiones de género y feminismos; luego se incluyen las temáticas de "cuerpo y salud", "cuerpo y percepción" y "cuerpo y resistencias políticas". 
Entre las temáticas abordadas en los talleres de lectura y encuentros de discusión del área, recuperadas a partir de su explicitación en las convocatorias a los eventos, pueden especificarse algunas diferencias entre los primeros años (2013-2016) y las inclusiones que se han integrado en estos últimos (2017-2019, hasta el período previo al inicio de la pandemia, cuando las convocatorias fueron interrumpidas). En la primera etapa, en las 24 convocatorias analizadas, como líneas de trabajo privilegiadas están marcos conceptuales para trabajar la corporalidad y los encuentros intercorporales, con privilegio de perspectivas fenomenológicas y postestructuralistas; cuerpo y género, cuerpo y relaciones sexo-afectivas; cuerpo y danza; cuerpos y diversidades funcionales; cuerpo e imagen.

En la etapa más reciente, de 20 convocatorias analizadas, si bien los ejes anteriores se mantienen, prevalece con fuerza notoria el de cuerpos y feminismos, que contempla cuestiones como las de diversidad sexual, corporal y funcional. Asimismo, se hace patente el interés por la corporalidad colectiva y por sus formas de expresión en diferentes períodos históricos, especialmente de la posdictadura argentina. En intersección con este último eje, a la vez, se potencian las discusiones en la línea de cuerpo, arte y política, especialmente en el cruce con cuerpo y danza. También, específicamente en las últimas dos convocatorias se ve un desplazamiento hacia la cuestión de cuerpo y salud.

A propósito de las monografias de los seminarios, cabe especificar que se analizaron dos cursadas implementadas en los segundos cuatrimestres de 2019 y de 2020, que la mayoría de los trabajos son grupales (elaborados entre dos, tres o cuatro personas, en general finalizando la carrera de grado) y que la elección temática de los mismos es libre, con la única condición de abordar la corporalidad en cualquiera de sus variantes; además, comprenden una extensión de entre 10 y 15 páginas promedio. Así las cosas, se han sistematizado las temáticas correspondientes a 70 trabajos entregados como monografías finales de la cursada.

A partir de esta sistematización, que apunta a mostrar el modo en que se hace presente el cuerpo en trabajos de comunicación, se han podido establecer cinco agrupamientos temáticos principales: diversidad corporal y feminismos (31 trabajos); cuerpos y afectos en protestas políticas (13 trabajos); cuerpo y arte (10 trabajos); cuerpo y vínculos sexo-afectivos (ocho trabajos); cuerpo y salud (ocho trabajos). La Figura 1 muestra esta distribución de un total de 70 monografias en los agrupamientos temáticos principales referidos. 
Figura 1. Temas de monografías (total de 70 trabajos).

Estos cinco ejes, no obstante, constituyen delimitaciones cuyos contenidos se entrecruzan. A continuación, la breve valoración de los contenidos de cada uno:

- Diversidad corporal y feminismos. Incluye 31 trabajos sobre temas como activismo gordo; ensayos críticos de las representaciones corporales en los medios (de cuerpos feminizados, migrantes, racializados, LGBTQI+ e intersex); representaciones de la corporalidad en las redes; cuerpo y vejez; estereotipos de género y constitución del cuerpo; normatividad corporal y corporalidades disidentes o abyectas; cuerpo, indumentaria y normatividades corporales; cuerpos y cuidados.

- Cuerpos y afectos en protestas políticas. Incluye 13 trabajos sobre redes afectivas en la protesta; sobre estéticas festivas como formas de comunicación; sobre la danza colectiva como estrategia de resistencia en manifestaciones; sobre la corporalidad colectiva en manifestaciones; sobre formas de hacerse presente el cuerpo en períodos históricos, sobre todo cuerpos feminizados durante y en la posdictadura.

- Cuerpo y arte. Incluye 10 trabajos, especialmente sobre cuerpo y danza; sobre la danza como forma de comunicación; sobre arte y percepción; sobre representaciones de los cuerpos feminizados en el terreno del arte.

- Cuerpo y salud. Incluye ocho trabajos en la línea de cuerpo, imperativos estéticos y salud; cuerpo y violencia obstétrica; cuerpo y aborto; cuerpo y alquiler de vientres.

- Cuerpo y vínculos sexo-afectivos. Incluye ocho trabajos sobre responsabilidad afectiva; sobre sexualidad y placer; sobre redes tecnopolíticas y lazos sociales entre los cuerpos; sobre cambios en los lazos afectivos con el contexto de la pandemia; sobre transformaciones en la subjetividad a partir de la sustracción del contacto corporal en el contexto actual de COVID-19. 
Finalmente, en cuanto a las elecciones temáticas de tesistas, se toman las solicitudes de tutoría que llegaron al Grupo de Investigación en Comunicación durante el segundo cuatrimestre del último año (2020): 13 en total. Aquí predominan elecciones relacionadas con diversidad corporal y sexual; representación del cuerpo y de configuraciones sexo-genéricas en medios masivos y redes sociales; análisis de la presencia del cuerpo en los discursos legislativos en relación con leyes de formación obligatoria en género y derechos reproductivos/aborto; redes de afecto, corporalidad colectiva y modalidades de la protesta en manifestaciones feministas; relaciones entre comunicación tecnopolítica y puesta en juego de los cuerpos en las formas de acción colectiva; cuerpo y danza; corporalidades y modos de producción en experiencias de arte-política.

\section{Reflexiones finales. Sobre avances, desplazamientos y desafíos}

Este artículo se propuso dar cuenta del cruce "cuerpo y comunicación" y de los aportes al campo de la comunicación que se vienen desarrollando en este terreno desde el análisis de un espacio institucional específico: el Área Cuerpo y Comunicación.

En lo expuesto puede observarse el crecimiento de la temática de la corporalidad en el espacio institucional estudiado, que corresponde a una universidad sumamente reconocida de la Argentina, cuyos desarrollos resultan de incidencia en el campo de la comunicación. La relevancia de la cuestión de la corporalidad y la diversificación temática, cada vez mayor, que incorpora el cruce "cuerpo y comunicación" resulta notoria en los últimos años. Esto pareciera mantener un estrecho nexo con el contexto de desplazamientos de las últimas décadas hacia una valorización de lo sensible y de la corporalidad y, sobre todo, con la agudización y masificación de los feminismos en los años recientes, que han puesto foco en el cuerpo como territorio político y han profundizado el interés por las problemáticas vinculadas.

Al respecto, por otro lado, en los años más recientes pueden hallarse algunos desplazamientos o la intensificación de preocupaciones en relación con la corporalidad en el espacio institucional estudiado. En la última etapa analizada, aproximadamente entre 2017 y 2020, se advierte un aumento pronunciado del interés por problemáticas y perspectivas feministas. Esto se ve tanto en la segunda etapa de las convocatorias del área como en las monografías de las últimas cursadas de los seminarios respecto de la primera etapa del área y respecto de las monografías presentadas en el Congreso Latinoamericano de Comunicación en 2015. 
Si bien presente en la primera etapa, en los años recientes ha crecido de forma notoria la inquietud por cuestiones feministas. Esto se expresa principalmente en el eje relativo a diversidad sexual y corporal, y a la inquietud por estudiarlo según preguntas rectoras de investigación y en distintos terrenos (como los medios masivos de comunicación, las redes sociales, etc.). Otra cuestión que ha cobrado importancia en este marco es la inquietud por estudiar las modalidades de encuentro y afecto entre cuerpos en las protestas feministas, considerándolas como formas de comunicación, y otros fenómenos relativos a la corporalidad colectiva, como los modos festivos de expresión en la protesta, las redes de comunicación en encuentros asamblearios, etc.

Progresivamente, así, las diferentes problemáticas e intereses sobre el cruce "cuerpo y comunicación" se manifiestan en la ampliación de espacios institucionales y de producciones teóricas, así como en el destacado aumento de la convocatoria de seminarios específicos. Ahora bien, aun cuando la apertura de estos espacios y la implementación de seminarios curriculares logran contener una parte importante del crecido interés sobre estos temas, uno de los principales desafios para su incorporación sistematizada tiene que ver con la posibilidad de incluir estas cuestiones en el núcleo curricular de grado como contenidos en las materias obligatorias, en las que no suele haber propuestas que aborden específica y sistematizadamente el cruce mencionado, independientemente de que puedan incluir (de modo mayormente disperso) lecturas o preguntas que aporten a la cuestión de la corporalidad.

La dificultad de incorporar las temáticas trabajadas en el área y en el seminario a un espacio curricular de contenido obligatorio reside, sobre todo, en la rigidez propia de los planes de estudio y en los procesos burocráticos necesarios para una modificación de los mismos. Estos procesos, incluso independientemente de la mayor o menor voluntad coyuntural de las funcionarias y los funcionarios responsables, resultan de por sí engorrosos e involucran instancias de la carrera, la facultad y la universidad en general. De ahí que no suelan responder a los tiempos que demandan procesos históricos vertiginosos como los de los últimos años y que se dificulte incorporar las inquietudes y posibilidades que se expresan en nuevas orientaciones de investigación a los contenidos obligatorios de grado.

Es por esto que las inquietudes emergentes sobre cuerpo en el campo suelen tratarse bajo propuestas de seminarios optativos, o en programas de seminarios de posgrado. El desafío presente está en pensar cómo estas cuestiones pueden ser incorporadas más sistemáticamente al grado y cubrir así esa nueva zona de preocupaciones en torno al cuerpo y la comunicación, que con tanta fuerza se impone en los intereses concretos de estudiantes, investigadoras e investigadores del campo. 


\section{Referencias}

Alcalá, V. (2015). La danza colectiva como política de visibilización. Revista Giró Cartelera. http:/ / www.girocartelera.com/columnas/escrituras-colectivas/la-danza-colectivacomo-politica-de-visibilizacion

Área Cuerpo y Comunicación. (2015). Una política de los cuerpos (Cuadernillo 1) [Issuu]. Carrera de Ciencias de la Comunicación, Facultad de Ciencias Sociales, Universidad de Buenos Aires. https: / / issuu.com/areadecuerpoycomunicacion/docs/cuadernillo_web_

Aschieri, P. y Puglisi, R. (2010). Cuerpo y producción de conocimiento en el trabajo de campo: una aproximación desde la fenomenología, las ciencias cognitivas y las prácticas corporales orientales (pp. 127-148). En S. Citro (Comp.), Cuerpos plurales. Antropología de y desde los cuerpos. Editorial Biblos.

Bardet, M. (2018). Saberes gestuales. Epistemologías, estéticas y políticas de "un cuerpo danzante". Revista Enrahonar. An International Journal of Theoretical and Practical Reason, 60, 13-28. https: / / dialnet.unirioja.es/ servlet/articulo? codigo=6357638

Braidotti, R. (2004). Feminismo, diferencia sexual y subjetividad nómade. Editorial Gedisa. Congreso Latinoamericano de Comunicación. (2015). Actas [PDF]. Carrera de Ciencias de la Comunicación, Facultad de Ciencias Sociales, Universidad de Buenos Aires. http:/ / comunicacion.sociales.uba.ar/wp-content/uploads/sites/16/2015/09/Libro-Congresode-Comunicacin-final.pdf

Butler, J. (1999). El género en disputa. Editorial Paidós-Pueg.

Butler, J. (2002). Cuerpos que importan. Editorial Paidós.

Cáneva, V. (2020). Prólogo. En M. Scarnatto y F. A. De Marziani (Comps.), Investigar en Cuerpo, Arte y Comunicación. Perspectivas e intersecciones en la producción de conocimiento. Teseopress.

Citro, S. (2010). Presentación. En S. Citro, Cuerpos plurales. Antropología de y desde los cuerpos. Editorial Biblos.

Federici, S. (2015). Calibán y la bruja. Mujeres, cuerpo y acumulación originaria. Tinta Limón Ediciones.

Fuentes, M. (2015). Performance, política y protesta. En D. Taylor y M. Steuernagel, ¿Qué son los estudios en performance? Hemi Press \& Duke University Press. https: / /scalar.usc. edu/nehvectors/wips/performance-poltica-y-protesta

Fuentes, M. (2020). Activismos tecnopolíticos. Constelaciones de performance. Eterna Cadencia Editora.

Gago, M. V. (2020). Lecturas sobre feminismo y neoliberalismo. Revista Nueva Sociedad, 290. https: / / nuso.org/articulo/lecturas-sobre-feminismo-y-neoliberalismo/

Haraway, D. J. (1995). Ciencia, cyborgs y mujeres. La reinvención de la naturaleza. Ediciones Cátedra.

Rizo García, M. (2018). Cuerpo y comunicación. Reflexiones teóricas y estado de la cuestión en México. Questión, Revista Especializada en Periodismo y Comunicación, 1(60), 1-15. https: / / perio.unlp.edu.ar/ojs/index.php/question/article/view/4737

Rubin, M. J. (2016). Contact Improvisación: el movimiento en constante presente. Revista Revol. http: / / revistarevol.com/actualidad/contact-improvisacion-el-movimiento-enconstante-presente/ 
Scarnatto, M. y De Marziani, F. A. (2020). Investigar en Cuerpo, Arte y Comunicación. Perspectivas e intersecciones en la producción de conocimiento. TeseoPress.

Scribano, A. (2016). La sociología de los cuerpos y las emociones en América Latina a través del GT26 de la Asociación Latinoamericana de Sociología ALAS [PDF]. Espacio Abierto, Cuaderno Venezolano de Sociología, 25(4), 159-168. https: / www.redalyc.org/ pdf/122/12249087012.pdf

Scribano, A. y De Sena, A. (2009). Construcción de conocimiento en Latinoamérica: algunas reflexiones desde la autoetnografía como estrategia de investigación. Cinta de Moebio, Revista de Epistemología en Ciencias Sociales, (34), 1-15. https://www.moebio.uchile. $\mathrm{cl} / 34 /$ scribano.html

Torres Cabreros, D. (2015, junio 16). Saltar, rolar, improvisar (La experiencia del Área de Cuerpo y Comunicación en Sociales). Página/12. https://www.pagina12.com.ar/diario/ universidad/10-275004-2015-06-16.html

Zilberman, J. (2015). 30 años de Contact en la Argentina [Issuu]. Balletin Dance, Revista Argentina de Danza, año 22, (246), 42-43. https: / / issuu.com/balletin/docs/ balletin_ dance_246 